\title{
Dissociating Timing and Coordination as Functions of the Cerebellum
}

\author{
Jörn Diedrichsen, ${ }^{1,2}$ Sarah E. Criscimagna-Hemminger, ${ }^{1}$ and Reza Shadmehr ${ }^{1}$ \\ ${ }^{1}$ Laboratory for Computational Motor Control, Department of Biomedical Engineering, Johns Hopkins University, Baltimore, Maryland 21218, and \\ ${ }^{2}$ Wolfson Center for Cognitive Neuroscience, School of Psychology, University of Wales, Bangor LL57 2AS, United Kingdom
}

The function of the cerebellum in motor control is a long-standing puzzle because cerebellar damage is associated with both timing and coordination deficits. Timing is the ability to produce consistent intervals between movements based on an internal representation of time. Coordination, in contrast, is a state-dependent control process in which motor commands to one effector depend on the predicted state of another effector. Here we considered a task consisting of two components, an arm movement and an isometric press with the thumb. We found that when the two components temporally overlapped, the brain controlled the thumb using an estimate of the state of the arm. In contrast, when the components did not temporally overlap, the brain controlled the thumb solely based on an internal estimate of time. Using functional magnetic resonance imaging, we contrasted these two conditions and found that lobule $\mathrm{V}$ of the cerebellum ipsilateral to the arm movement was consistently more activated during state-dependent control. When the brain learned time-dependent control, no region of the cerebellum showed consistently increased activity compared with state-dependent control. Rather, the consistent activity associated with time-dependent control was found in language areas of the left cerebral hemisphere along the Sylvian fissure. We suggest that timing and coordination are behaviorally distinct modes of motor control and that the anterior cerebellum is a crucial node in state-dependent motor control, computing a predictive state estimate of one effector (e.g., the arm) to coordinate actions of another effector (the thumb).

Key words: motor control; fMRI; timing; forward models; generalization; cerebellum

\section{Introduction}

It is unclear whether deficits after cerebellar damage are best characterized as deficits in timing or coordination (Mauk et al., 2000). On the one hand, patients with cerebellar lesions exhibit increased temporal variability in the production of periodic but discontinuous movements, such as simple finger tapping with an interleaved pause (Ivry and Keele, 1989; Spencer et al., 2003). The same patients are also impaired in judging temporal intervals between sensory stimuli (Ivry and Keele, 1989), a deficit that is thought to account for the impairment in eyeblink conditioning (Gerwig et al., 2003). When a task has discrete components, control may rely on an explicit representation of the time between these, here called time-dependent control, and the cerebellum may be critical for this representation (Ivry et al., 2002).

However, cerebellar patients often show deficits, even when movements do not consist of discrete components. During reaching, cerebellar patients fail to compensate for interaction torques that arise between shoulder and elbow joints (Bastian et

\footnotetext{
Received Jan. 7, 2007; revised April 24, 2007; accepted April 25, 2007.

This work was supported by grants from the National Institutes Health (NIH) (NS37422), the Human Frontiers Science Program, the Johns Hopkins General Clinical Research Center (RPN 02-08-15-03), and the Biotechnology and Biological Sciences Research Council (BB/E009174/1). We are grateful to the staff of the F.M. Kirby Research Center for Functional Brain Imaging at Kennedy Krieger Institute, funded by an NIH/National Center for Research Resources resource grant (RR15241), and to Richard lvry and Amy Bastian for helpful comments.

Correspondence should be addressed to Jörn Diedrichsen at the above address. E-mail: j.diedrichsen@ bangor.ac.uk.

D01:10.1523/JNEUROSC1.0061-07.2007

Copyright $\odot 2007$ Society for Neuroscience $\quad$ 0270-6474/07/276291-11\$15.00/0
}

al., 1996). When throwing a ball, they fail to coordinate the opening of the hand with the movement of the upper arm (Timmann et al., 1999). In healthy individuals, movements of this kind are coordinated using a state-dependent control. For example, during throwing, the opening of the fingers for ball release depends on an estimate of the position of the arm (Hore and Watts, 2005). Cerebellar deficits may arise because the healthy cerebellum contributes to state-estimation through a forward model of limb dynamics (Wolpert et al., 1998).

Because time- and state-dependent control both produce temporally precise motor commands, it seems difficult to differentiate these two ideas (Mauk et al., 2000). One critical difference, however, lies in the way a skill generalizes. For example, Conditt and Mussa-Ivaldi (1999) trained people to reach while holding a tool that produced time-dependent forces. Participants successfully adapted their motor commands. However, the pattern of generalization showed that they had learned state- and not timedependent control: they generalized the skill to circular movements, requiring a temporal sequence of motor commands that they had never experienced. In other tasks, however, participants appear to acquire the skill by learning the temporal relationship between events (Karmarkar and Buonomano, 2003). For example, Meegan et al. (2000) trained participants to discriminate either 300 or $500 \mathrm{~ms}$ intervals, and found that this training improved the production of the trained but not the untrained interval. Here, motor commands depended on a central representation of time, shared across task domains (Ivry, 1996). 
What determines whether control is time- or statedependent? Here we developed a paradigm that mimics the coordination demands between the arm and fingers during reachto-grasp movements. We taught participants to execute two actions, a point-to-point arm movement and an isometric force pulse with their thumb (see Fig. 1A). By regulating the relative timing of these actions, we manipulated whether participants relied on state- or time-dependent control. We then used this behavioral dissociation in a functional imaging study to examine the contribution of the cerebellum to the coordination of the actions.

\section{Materials and Methods}

Participants. Sixty participants (33 male, 27 female) were recruited for experiment 1 (average age, 27 years; SD, 7 years). Five individuals were left handed, and all participants performed the task with their right hand. Eleven new right-handed participants (five male, six female) participated in experiments 2 and 3 (average age, 25 years; SD, 2.6 years), and 11 new right-handed participants (five male, six female) were recruited for experiment 4 (average age, 23 years; SD, 2.4 years). All procedures were approved by the Johns Hopkins School of Medicine Institutional Review Boards.

Apparatus. Participants made reaching movements using a two-joint robotic manipulandum, which restricted movements to the horizontal plane and was capable of applying forces to the hand. Position and velocity of the hand were recorded at $100 \mathrm{~Hz}$. In experiments 1 and 2, participants sat in front of the robot while viewing a vertical computer monitor. The starting and target boxes (each $2.5 \times 2.5 \mathrm{~cm}$ ) were presented on the screen arranged horizontally at a distance of $8 \mathrm{~cm}$ (see Fig. $1 B)$. A $5 \times 5 \mathrm{~mm}$ cursor provided continuous visual feedback of the hand position.

The thumb press was recorded by an isometric load cell that could be depressed with the thumb (accuracy, $0.02 \mathrm{~N}$; max force, $40 \mathrm{~N}$ ). Participants were instructed to keep the thumb placed on the load cell at all times, such that a continuous force reading was available. In experiment 1, the load cell was mounted on the robot handle (see Fig. $1 A$ ), in experiments 2 and 3, a separate handle with the load cell was held in the left hand, while the participants moved the robot with their right hands.

For functional imaging (experiment 3), the bilateral task was performed in the supine position. We used a functional magnetic resonance imaging (fMRI) compatible robotic device with optical encoders and a nonmagnetic force sensor (Diedrichsen et al., 2005). All cables were shielded and filtered to avoid leakage of radio frequency into the scanning room. Tests established that these devices did not increase the noise level in the magnetic resonance signal.

Experiment 1. Participants were trained to execute an $8 \mathrm{~cm}$ point-topoint arm movement with a movement time (MT) of $350 \mathrm{~ms}$. Each trial began with the cursor in the starting box (see Fig. $1 B$ ). A red target appeared and turned white after a variable interval of $0.5-1 \mathrm{~s}$. The start of the arm movement was defined as the first time the forward hand velocity exceeded $3.5 \mathrm{~cm} / \mathrm{s}$. The end of the movement was defined as the first time when the forward velocity dropped $<3.5 \mathrm{~cm} / \mathrm{s}$. The difference between the produced and the targeted movement time was indicated by an arrow to the right of the target. The color and direction of the arrow specified whether the arm moved too slow (blue) or too fast (red), with the length of the arrow indicating the magnitude of the error. Participants were instructed to make the arrow as short as possible. Pretraining consisted of 60 trials in which only the reach was performed, the first half had a targeted movement time of $350 \mathrm{~ms}$, whereas the second half had a targeted movement time of $550 \mathrm{~ms}$.

Participants were then instructed to produce a short force pulse with the thumb before, during, or after the movement. The critical taskrelevant variable was $\Delta T$, the time interval between the time of peak force of the thumb and the start of the arm movement $\left(T_{\text {peak force }}-T_{\text {movement }}\right.$ start), which calculated as a measure of the relative timing of the two movement components (see Fig. 1C). Participants were assigned to one of eight groups with a specific target interval, $\Delta T=-500,-250,-150$, $-100,50,150,250$, and $350 \mathrm{~ms}$, or to one of the two control groups (see below). If the arm movement time was within $65 \mathrm{~ms}$ of the goal of $350 \mathrm{~ms}$, feedback was provided to indicate the relative timing $(\Delta T)$ of the two components. An ellipse appeared together with three lines (see Fig. $1 B$ ). If the ellipse appeared above the middle line, peak force had occurred too late. If the ellipse appeared below the middle line, peak force had occurred too early. Participants were simply instructed to produce arm movement and thumb press and to change the temporal relationship to get the ellipse onto the middle line. The scaling of the feedback was adjusted after every block depending on the accuracy of the participant. After each block, participants also received a score denoting their average performance on the task.

Participants were trained in six blocks consisting of 60 trials each (see Fig. $1 D$ ), from which the last two constituted the baseline phase. We then tested how participants generalized the skill to a situation in which the arm movement was slower ( $550 \mathrm{~ms}$ instead of $350 \mathrm{~ms}$ ). In the spontaneous generalization block (20 movements), we provided feedback only about the speed of the arm movement, but not about $\Delta T$. These trials measured how participants naturally generalized the task. To avoid any bias toward either timing or scaling strategy, participants received an ambiguous instruction concerning the thumb press: "You will now perform the learned skill with a slower movement. You will not receive any feedback about your thumb press, but press the button as you have in the previous training blocks." During the slower movements, we shifted the starting arm position and target position forward by $5 \mathrm{~cm}$.

If participants learned to perform the task by controlling the time between the onsets of the two components (time-dependent control), slowing down the arm movement should not impact this temporal representation. Therefore, $\Delta T$ or any other characteristic of the thumb press should remain unchanged (see Fig. $1 E$, top). If, however, participants learned the task using a state-dependent control, we should see predictable changes in the timing and duration of the thumb press. The exact changes depend on the state variable that is used to coordinate the two components. Because the absolute velocity and the position of the hand were changed in the generalization phase, neither of these two state variables could be used alone. We therefore hypothesized that people would use a more abstract state representation that approximates the percentage of movement completed. Under this assumption, the temporal gap between peak thumb force and arm movement start $(\Delta T)$ should scale proportionally with the arm movement time. Furthermore, because the start and end of the thumb press also occur at a certain state of the arm movement, the duration of the thumb press should also become proportionally longer (see Fig. $1 E$, bottom). To calculate the predicted $\Delta T$ and the duration of the thumb press in the spontaneous generalization block, we calculated a movement time scaling factor $\left(\mathrm{MT}_{\text {test }} / \mathrm{MT}_{\text {baseline }}\right)$ for each participant. This factor was multiplied with the $\Delta T$ or the duration of the thumb-press in the last two blocks of training. This resulted in the predicted change of these two variables across the groups (see Fig. $2 A, B$, gray line). The predicted change is slightly irregular, because not all participants achieved the full movement time of $550 \mathrm{~ms}$ (mean MT, $515 \mathrm{~ms}$; $\mathrm{SD}, 32 \mathrm{~ms}$ ). We then performed one-sample $t$ tests for each group to test for a difference between observed and predicted values under the stateand time-dependent control hypotheses.

Because the results of the spontaneous generalization may depend on how exactly participants understood the ambiguous instruction ("Move slower and press the button as in training"), we tested their performance in two transfer tests (see Fig. 1D, 48 movements each). Here, the slow movement had to be performed with a particular goal for $\Delta T$, and feedback was given as in training. Unbeknown to the participants, the goal for $\Delta T$ was either the same (absolute transfer) or scaled proportionally with the movement time goal (proportional transfer). For example, if participants were trained to produce a $\Delta T$ of $250 \mathrm{~ms}$, the new goal could either stay $250 \mathrm{~ms}$ (absolute transfer) or become $393 \mathrm{~ms}$ (proportional transfer). We predicted that participants who learned the task using the timedependent control should perform better during absolute transfer, whereas participants who learned the task using the state-dependent control should perform the task better during the proportional transfer.

After the first transfer block, participants performed another block of training with the original fast movement speed (60 movements), followed by a second spontaneous generalization test and a transfer block. 
Half of the participants were tested with absolute transfer first, followed by proportional transfer; for the other half, the sequence was reversed.

To test two alternative hypotheses for the generalization result found in experiment 1, we included two control groups. The first group was included to test the hypothesis that the feedback on $\Delta T$, which was given in terms of absolute time, had biased participants toward using timedependent control. For this group $(\Delta T=-318 \mathrm{~ms})$ feedback on $\Delta T$ was given as percentage of the movement time produced during that trial (i.e., for a slower movement, a longer $\Delta T$ had to be produced to get the same feedback). In the second control group, we tested the hypothesis that temporal order rather than temporal overlap determined whether the button press was scaled with the arm movement. This group was trained to produce a thumb press $350 \mathrm{~ms}$ after the end of the movement. In this condition, feedback was based on the interval from movement end to the time of maximal thumb force. If temporal overlap rather than the order of the components was the determining factor for the influence of the arm movement on the thumb, then producing a thumb press after the movement should lead to the same result as producing the thumb press before the movement.

Experiment 2. We used a bilateral version of the task for the fMRI experiments (experiments 3 and 4). Participants moved one arm while producing the press with the thumb on the contralateral hand. By using this design, the neural activity related to the arm movement and the thumb press were spatially separated across hemispheres. In experiments 2 and 3, subjects moved the right arm and pressed the left thumb. In experiment 4 , the pattern was reversed. To establish that the results from the unilateral task (experiment 1) generalized when the task was bilateral, we trained participants in two separate sessions in the bilateral task. In one training session, they learned to produce a $\Delta T$ of $-350 \mathrm{~ms}$ (nonoverlapping condition), and in one training session they learned to produce a $\Delta T$ of $+350 \mathrm{~ms}$ (overlapping condition). Each of these sessions was identical to experiment 1 , consisting of pretraining, $6 \times 60$ training trial, 20 spontaneous generalization trials, 48 transfer test trials (either proportional and absolute), and 60 trials of intermediate training, followed by a second transfer test (see Fig. 1D). All participants that participated in experiment 2 then underwent fMRI (experiment 3 ).

Experiment 3. During imaging, participants did not return to the starting box, but executed arm movements in both directions. This avoided additional activation caused by the passive return to the starting box. The targets were arranged such that the movements mostly involved the elbow joint. Participants performed four conditions in a block design: thumb-only, arm-only, the overlapping, and the nonoverlapping condition. In the thumb-only condition, participants were instructed to produce a force pulse with their left thumb each time a new target appeared. An arrow on the left provided feedback on their maximal force, pointing upward if they were $<18 \mathrm{~N}$ and pointing downward if they were $>18 \mathrm{~N}$. In the arm-only condition, subjects made a right arm movement and feedback on the movement time was displayed as an arrow on the right, indicating the deviation from $350 \mathrm{~ms}$. In the overlapping and nonoverlapping conditions, feedback about the timing of the button press (too late vs too early) was given as an arrow on the left and feedback on the movement time was given as an arrow on the right.

A fixation cross was presented in the center of the display. Participants were instructed to try to maintain fixation at all times. The targets and feedback subtended a visual angle of $4.5^{\circ}$ and could be clearly seen even when maintaining fixation. The size of the visual feedback error was scaled such that it was on average of the same size in the overlapping and nonoverlapping conditions. To test how well participants could maintain fixation, we performed an additional control experiment with six participants who performed the same task outside of the scanner while we monitored eye movements. Participants made an average of only 0.33 saccades per trial ( 0.17 in button-only, 0.23 in arm-only, 0.54 in the nonoverlapping, and 0.40 in the overlapping condition).

The task was performed in a blocked design. At the beginning of each block, the word "button," "move," "button-move," or "move-button" was presented for $2 \mathrm{~s}$ to indicate the upcoming condition. Six movements (three times back and forth) were performed in the given condition. Each trial lasted $4 \mathrm{~s}$ and each block of six trials was followed by a $6-8 \mathrm{~s}$ pause. After each block, feedback was given on overall performance and partic- ipants were verbally informed if they did not match the maximal force of the thumb press, the length of the thumb press, or the movement time between conditions accurately. Before imaging, participants were trained on this paradigm lying down in a "mock scanner" for $1 \mathrm{~h}$ to familiarize them with the task.

Experiment 4. A second set of participants was recruited to perform a variant of experiment 3: participants performed the arm movement with the left arm and the button press with the right hand. This allowed us to test whether the lateralization of the findings were because of left-right hemispheric asymmetries or because of assignment of the actions to the hands. This experiment also included a second nonoverlapping condition, in which the sequence of thumb press and arm movement was reversed. Therefore, in experiment 4, we could compare the overlapping condition to the average of the two nonoverlapping conditions, balancing for the influence of the sequence of the two actions.

Participants performed five conditions in a block design: arm only, thumb only, the overlapping condition ( $\Delta T$ of $350 \mathrm{~ms})$, the nonoverlapping condition with thumb first ( $\Delta T$ of $-350 \mathrm{~ms}$ ), and the nonoverlapping condition with the arm first. The latter condition was similar to control condition 2 in experiment 1, in that feedback was given on the temporal gap between the end of the arm movement and the maximal thumb force, with a goal of $350 \mathrm{~ms}$, resulting in an effective $\Delta T$ of $700 \mathrm{~ms}$. Pretraining in each of these condition was performed as described in experiment 2, with the order of these trainings counterbalanced between participants. However, no spontaneous generalization and transfer test were acquired. Participants then practiced the task in a supine position for $1 \mathrm{~h}$ and finally underwent fMRI scanning.

Imaging acquisition. Data were acquired on a 3T Philips Intera system (Philips Medical Systems, Best, The Netherlands). For functional scans, we used an echoplanar imaging sequence with sensitivity-encoded MRI (Pruessmann et al., 1999) and a SENSE-factor of 1 . We used 31 slices (3 $\mathrm{mm}$ thickness; $0.2 \mathrm{~mm}$ gap; repetition time, $2 \mathrm{~s}$ ) in a $45^{\circ}$ oblique angle to cover the cortical motor areas and the cerebellum. Therefore, we did not record data from the inferior aspects of the prefrontal cortex or from the anterior temporal lobes. Each image was acquired as an $80 \times 80$ matrix (field of view, $24.0 \times 24.0 \mathrm{~cm}$ ), with a voxel size of $3 \times 3 \times 3.2 \mathrm{~mm}$. The image was reconstructed to $128 \times 128$. Each scan consisted of four dummy images that were discarded and 154 data images. T1-weighted structural images were acquired with $1 \times 1 \times 1 \mathrm{~mm}$ resolution using an magnetization-prepared rapid-acquisition gradient echo sequence without sensitivity encoding for a higher signal-to-noise-ratio.

Data analysis. The behavioral velocity and force data were up-sampled to $200 \mathrm{~Hz}$ and smoothed with a Gaussian kernel of $20 \mathrm{~ms}$ width. Three temporal landmarks were determined for the force pulse: when the force first exceeded $5 \%$ of the peak force, when it reached peak force, and when it fell to $<5 \%$ of the peak force. A similar analysis was performed on the arm movement using the velocity in the direction of the target and a start/stopping criterion of $3.5 \mathrm{~cm} / \mathrm{s}$.

The functional data were analyzed using Matlab and SPM2 (http:// www.fil.ion.ucl.ac.uk/spm/) (Friston et al., 1994). First, we corrected for the temporal offset of slice acquisition and then spatially aligned the data to the first scan using a six parameter rigid-body transform. Data were high-pass filtered with a cutoff frequency of $1 / 128 \mathrm{~s}$ to remove slowly varying trends. The time series for each voxel was modeled using multiple regression with a separate regressor for each task phase. The regressors were generated by convolving a boxcar function spanning a block of six movements with a standard hemodynamic response function. One additional regressor per scan was used to model the neural response to the instruction stimulus. To control for possible noise artifacts in the data, we used a weighted least-squares approach that down-weights the images with higher noise variance (Diedrichsen and Shadmehr, 2005). The resulting coefficient estimates for each task block were then transformed into percentage of signal change by dividing the peak of the predicted response by the mean signal intensity for a given voxel. From this, the average percentage signal for each condition was computed.

Previous studies on coordination have sometimes relied on a contrast between the sum of the activity of two movement components ( $A$ and $B$ ) compared with a coordination condition (C) (Ramnani et al., 2001; Wenderoth et al., 2005). Each of the components is contrasted to rest 
$(R)$, yielding the effective contrast of $(A-R)+$ $(B-R)<C-R$; therefore [assuming linearity of blood oxygenation level-dependent (BOLD) signal], the effective contrast was $A+B<C+$ $R$. From this, it becomes immediately clear that the regions with a relatively high rest activity are more likely to become significant, resulting in a potentially biased result. Indeed, regions that were found in this contrast in the above studies showed negative activations of the movement components compared with rest. For example, in a study by Ramnani et al. (2001), coordination-related activity was found in the left cerebellum, although the task was performed with the right hand.

To avoid this bias, we relied here only on the direct comparison between the overlapping and nonoverlapping conditions. In experiment 4 , we compared the overlapping condition to the average activation of the two nonoverlapping conditions. To determine the influence of sequence effects, we also compared the two nonoverlapping conditions against each other. The arm-only and thumb-only conditions served to spatially localize the activity caused by movement components, but no direct comparisons to the overlapping and nonoverlapping conditions were made.

To reduce the impact of anatomical variability across subjects, we used three different strategies to average the data for group analysis. We isolated the cerebellum and brainstem in each subject and then matched these to a newly developed atlas template for infratentorial structures (Diedrichsen, 2006). The atlas template is spatially unbiased with respect to the MNI152 template while preserving the fine anatomical detail of the cerebellum. Alignment to this template using nonlinear deformations with a high resolution (cutoff frequency, 1 $\mathrm{cm}^{-1}$ ) significantly increases the overlap of functionally equivalent regions in the cerebellum. In the cerebellum, we tested differences between the percentage of signal change images with a planned $t$ test, applying an uncorrected threshold of $p<0.001, t_{(10)}=4.14$. We corrected for multiple comparisons over the cerebellar search volume by using corrected cluster-wise $p$ values, derived from Gaussian field theory (Friston et al., 1994).

For activity in the neocortex, we reconstructed the cortical surfaces of both cerebral hemispheres for each participant and projected their functional data onto it using caret software (http://brainmap.wustl.edu/resources/caretnew.html) (Van Essen et al., 2001) . Using six landmarks and spherical alignment, these surfaces were then brought into the population average landmark and surface-based atlas (Van Essen, 2005). We then used custom Matlab functions to correct for multiple comparisons on the surface (http://www.bangor.ac.uk/ pss412/imaging/surface stats.htm) (Diedrichsen, 2006) using a corrected $p$ value for the cluster size at an uncorrected height threshold of $p<0.002, t_{(10)}=3.72$. For the remaining subcortical areas, we used the nonlinear normalization to the MNI template (Ashburner and Friston, 1999). For all comparisons, we restricted the search region to areas that were positively activated compared with rest in at least one of the task conditions.

\section{Results}

\section{Experiment 1}

Participants were trained to produce a thumb press at various time points before, during, or after a $350 \mathrm{~ms}$ arm movement (Fig. $1 C)$. Ten groups of participants were run, each trained with a different $\Delta T$ as a goal. All groups learned to perform the task and improved over the course of six training blocks. The absolute
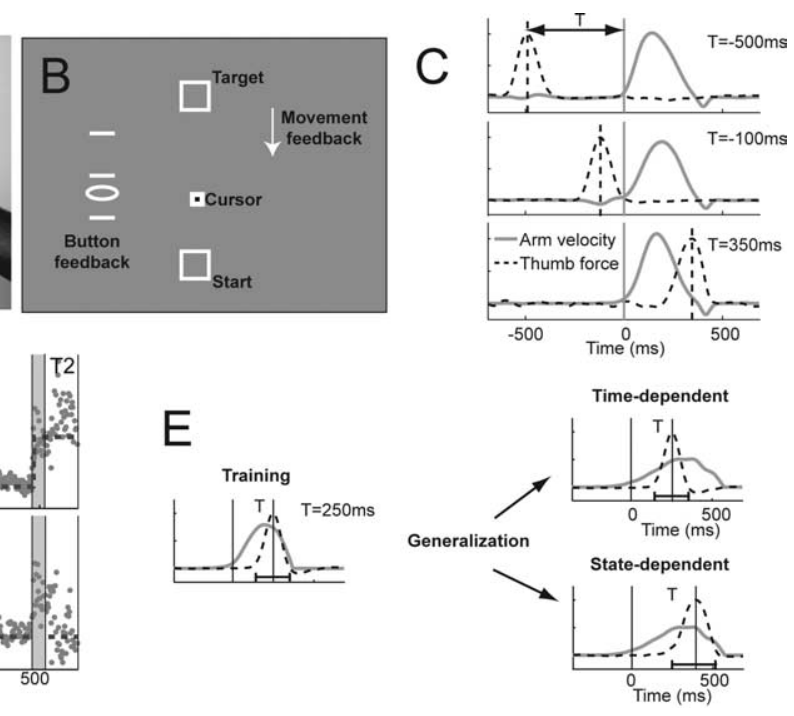

rial

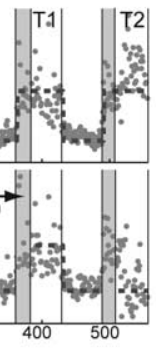

Figure 1. Unilateral thumb-arm task (experiment 1). $\boldsymbol{A}$, Participants held the handle of a robotic arm and placed their thumb on (dashed line) in a specific temporal relationship to movement start $(\Delta T)$. D. Exemplary data of one participant in the $\Delta T=350$ 列 to a slower movement. State-dependent control predicts that $\Delta T$ and the duration of the thumb press (black line) scale proportionally with movement time. Time-dependent control predicts that $\Delta T$ and the length of the thumb press do not change.

error of $\Delta T$ decreased by $45 \%$ from the first to the last training block across all participants.

Spontaneous generalization. To determine whether participants learned to produce the thumb press based on a representation of time or of arm state, we examined how the skill generalized when participants were instructed to make a slower arm movement. In the spontaneous generalization phase (Fig. 1D), participants were instructed to produce a $550 \mathrm{~ms}$ reaching movements and to "press the button as they learned in training," without receiving feedback regarding the timing of the thumb press.

When using the state-dependent control, the brain learns to coordinate the two components by making the motor commands to the thumb depend on the state of the arm (i.e., its position, velocity, or angle of joints). Because both position and peak velocity of the movement changed in the generalization phase, we hypothesized that participants would use a high-level combined representation that represents the percentage of movement completed. In this case, two specific changes for the thumb press during spontaneous generalization can be predicted. First, because the brain learned to produce peak thumb force at a particular state of the arm, $\Delta T$ (the relative timing between thumb and arm) should scale proportionally with the movement time (Figs. $1 E, 2 A$, gray line) (see Materials and Methods). Second, because the onset and offset of the thumb press would also be produced at a certain state of the arm, the duration of the thumb press, a feature of the response that was unconstrained and about which no feedback was given, should also increase proportionally with the arm movement time (Fig. $2 B$, gray line).

In contrast, when using a time-dependent control, the brain learns the task by accurately representing the absolute time between the onsets of the components. Thus, slowing the arm 

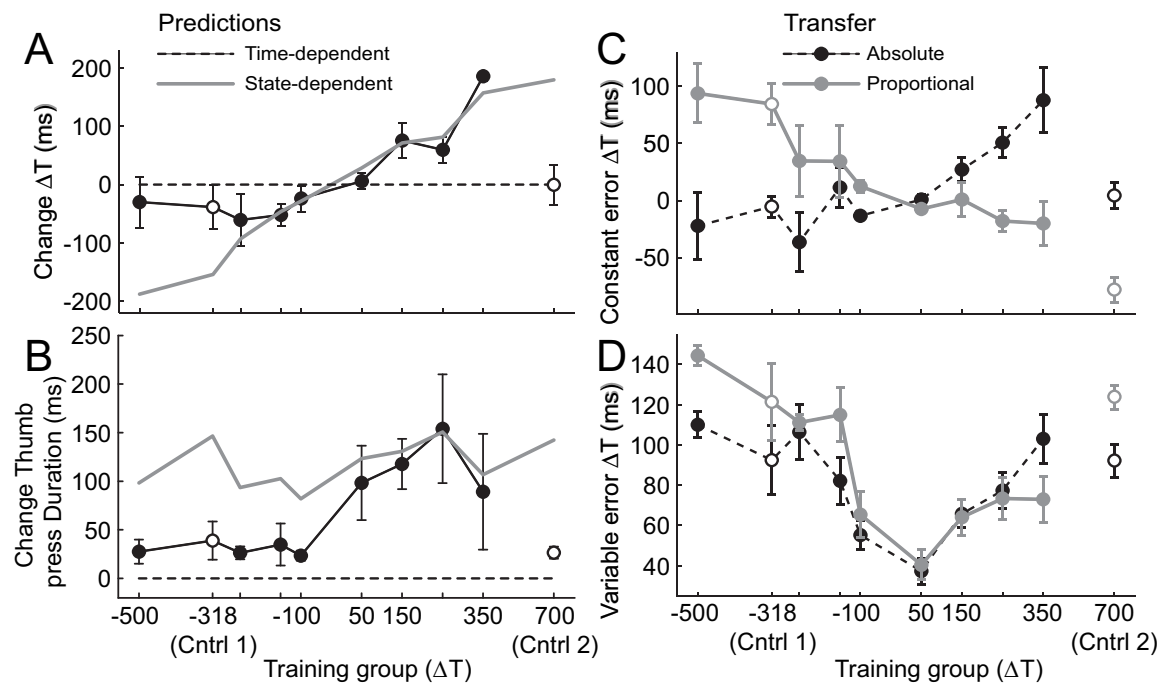

Figure 2. Results of the unilateral coordination task (experiment 1) for 10 groups of participants, each trained on a different temporal interval between movement onset and thumb press $(\Delta T)$. Results for the two control groups (see text) are shown in white. $\boldsymbol{A}, \boldsymbol{B}$, Change in $\Delta T(\boldsymbol{A})$ and change in the duration of thumb press $(\boldsymbol{B})$ from the last two training blocks to the first spontaneous generalization test. The gray line indicates the predicted change under the state-dependent control hypothesis, and the dashed line predicted the change under the time-dependent control hypothesis. $\boldsymbol{C}, \boldsymbol{D}$, Constant $(\boldsymbol{C})$ and variable error (SD; $\boldsymbol{D})$ of $\Delta T$ in the absolute and proportional transfer tests. Error bars indicate SEM. Cntrl, Control.

movement should not result in a change of $\Delta T$ (Figs. $1 E, 2 A$, dashed line) or of the duration of the thumb press (Fig. $2 B$, dashed line).

Our results suggest a transition from state- to time-dependent control as a function of the temporal overlap between the two task components. When the components overlapped, we observed that changing the speed of the arm movement affected the timing of the thumb press. In the 150, 250, and $350 \mathrm{~ms}$ groups (Fig. 2A), $\Delta T$ changed as predicted by the state-dependent control hypothesis. All one-sample $t$ tests against the prediction of time-dependent control were significant $\left(t_{(5)}>2.48 ; p<0.05\right)$. Additionally, the duration of the thumb press (Fig. $2 B$ ) was significantly prolonged in the groups with $\Delta T$ between +50 and $+350 \mathrm{~ms}\left(\right.$ all $\left.t_{(5)}>2.57 ; p<0.05\right)$.

When the two components did not overlap $(-500 \mathrm{~ms})$, the change in $\Delta T$ was consistent with the prediction of timedependent control and differed from the prediction of statedependent control $\left(t_{(5)}=2.71 ; p=0.04\right)$. Also, the change in the duration of the thumb press (Fig. $2 B$ ) conformed better with the prediction of time-dependent control.

In the transition area between state- and time-dependent control ( -250 to $-100 \mathrm{~ms})$, the results are less clear, possibly indicative of a mixture of control strategies. Participants did not prolong the thumb press as predicted by the state-dependent control hypothesis $\left(t_{(5)}>4.2 ; p<0.008\right)$ indicative of time-dependent control. In contrast, for the $-150 \mathrm{~ms}$ group, we found a significant change in $\Delta T$, congruent with the state-dependent control. For the -100 and +50 ms groups, the predictions in terms of $\Delta T$ were too similar to dissociate the hypothesis.

Apart from this transition area, however, the results are clear. When the two movement components overlapped, participants learned state-dependent control; temporal scaling of one movement resulted in temporal scaling of the other. When the two movement components were separated by even a small temporal gap, they learned time-dependent control, regulating the time between the onsets or offsets of each component.

\section{Control conditions}

We considered alternative explanations for the finding that participants used timedependent control for nonoverlapping components. First, the feedback in the main experiment was based on the absolute time between peak force of the thumb press and the start of the arm movement. This may have biased participants toward using the time-dependent control. We therefore introduced a control condition in which the feedback about $\Delta T$ was given as percentage of the movement time on that trial. That is, on trials with slower movements, $\Delta T$ had to be scaled correspondingly to achieve optimal performance. The group was trained on a temporal gap of $-318 \mathrm{~ms}$ (Fig. 2, white circle at $-318 \mathrm{~ms}$ ). Despite this manipulation, which favored a state-dependent mode of control, the generalization pattern showed time-dependent control.

Second, it is possible that the order of movement components rather than their temporal overlap determined the pattern of results. That is, perhaps the thumb press would scale with the arm movement if it were performed after the arm movement. To address this possibility, a second control group was trained to produce a thumb press $350 \mathrm{~ms}$ after the end of the movement. In this case, feedback was based on the time between the end of the arm movement and the time of maximal force. The results of this control condition (Fig. 2, white circle at $700 \mathrm{~ms}$ ) showed a pattern indicative of time-dependent control. Thus, the overlap of the two movement components rather than their order was the determining factor in whether control was time or state dependent.

\section{Transfer test}

We recognized that our results from the spontaneous generalization test might have depended on the participant's interpretation of the ambiguous instruction, "move slower and press the button as you did in training." To test whether the consequences of the training would also be visible if participants transferred to a new learning condition, we included two transfer tests (Fig. 1D) in which participants performed a $550 \mathrm{~ms}$ movement and received explicit feedback about $\Delta T$. If participants had learned statedependent control during training (Fig. $1 D$, trials 1-330), then they should exhibit a smaller systematic and variable error in $\Delta T$ in the proportional transfer test, during which the target $\Delta T$ was scaled with the movement time, and the thumb press had to be produced at a similar state of the arm. In contrast, if participants had learned time-dependent control, they should perform better during the absolute transfer test, during which the target $\Delta T$ was the same as in training.

The constant error in $\Delta T$ (Fig. $2 C$ ) showed transfer congruent with the results from spontaneous generalization. For conditions in which the thumb press preceded or occurred after the movement, $\Delta T$ showed a high constant error in the proportional transfer test (Fig. 2C, gray line) but was accurate for the absolute transfer test. For conditions in which the two components overlapped, $\Delta T$ showed a high constant error in the absolute transfer test (Fig. $2 C$, dotted line). The group by transfer interaction was highly significant $\left(F_{(9,39)}=24.32 ; p<0.001\right)$, and the pattern 
remained significant even if we excluded the first 20 movements of the transfer blocks. The variable error (Fig. 2D) was lowest in the $50 \mathrm{~ms}$ group, resulting from the fact that the SD of temporal intervals usually scales proportionally with the mean (Gibbon et al., 1997). Most importantly, each group also showed a higher variable error for the transfer condition in which they showed a higher constant error $\left(F_{(9,39)}=5.67 ; p<0.001\right)$.

In conclusion, the results from the explicit transfer corroborated the results for the spontaneous generalization test. Participants learned to coordinate movement components using a state estimate of the arm when the two components overlapped. However, when the components were separated by a temporal gap, the coordination depended on an internal measure of time.

\section{Experiment 2}

To establish that the results from experiment 1 would generalize to the bilateral task, we trained participants on separate days in the overlapping $(\Delta T=+350 \mathrm{~ms})$ and the nonoverlapping conditions $(\Delta T=-350 \mathrm{~ms})$, including the spontaneous generalization and transfer test on each day.

As in the unilateral experiment, participants scaled $\Delta T$ in the overlapping condition, whereas this scaling was absent in the nonoverlapping condition (Fig. 3A). For the transfer test (Fig. $3 B, C)$, performance in the overlapping condition was best in the proportional transfer test and in the nonoverlapping condition in the absolute transfer test. As predicted, the condition by transfer interaction was significant for constant error $\left(F_{(1,10)}=21.70 ; p<\right.$ $0.001)$ and for the variable error of $\Delta T\left(F_{(1,10)}=7.14 ; p=0.023\right)$. Therefore, regardless of whether the task was unilateral or bilateral, temporal overlapping of task components led to the statedependent control, whereas nonoverlapping led to the timedependent control.

\section{Experiment 3}

In experiments 3 and 4, we looked for the neural correlates of time- and state-dependent control. In experiment 3, the volunteers used their right arm for reaching and the left thumb for pressing. All participants performed the overlapping and the nonoverlapping conditions, as well as the individual actions, in isolation. By using a bilateral task, we could assess the exact role of the cerebellum in state-dependent coordination. If the cerebellum was involved in producing an accurate state estimate of the arm, then coordination-related activity during the overlapping condition should be found in the right cerebellum, ipsilateral to the arm movement. If, in contrast, the cerebellum received a state estimate of the arm and adjusted the motor commands to the thumb accordingly, then this activity should be found in the left cerebellum, ipsilateral to the thumb press.

\section{Behavioral results}

As in all fMRI studies of motor control, it is important that the comparisons are made between conditions that are comparable in movement parameters because small changes in movement speed or force can drastically alter the BOLD signal in movementrelated areas (Seidler et al., 2004). The duration of arm movement during the overlapping and nonoverlapping conditions was very similar (Table 1$)\left(t_{(10)}=0.88, p=0.39\right)$. Also, the total impulse for the thumb press (force $X$ time) (Table 1) was well matched $\left(t_{(10)}=0.40 ; p=0.694\right)$. The mean $\Delta T$ showed that participants could perform the overlapping and nonoverlapping conditions as instructed in the scanner. Although we did not perform the generalization test again in the scanner to ensure that participants used time- and state-dependent control to perform
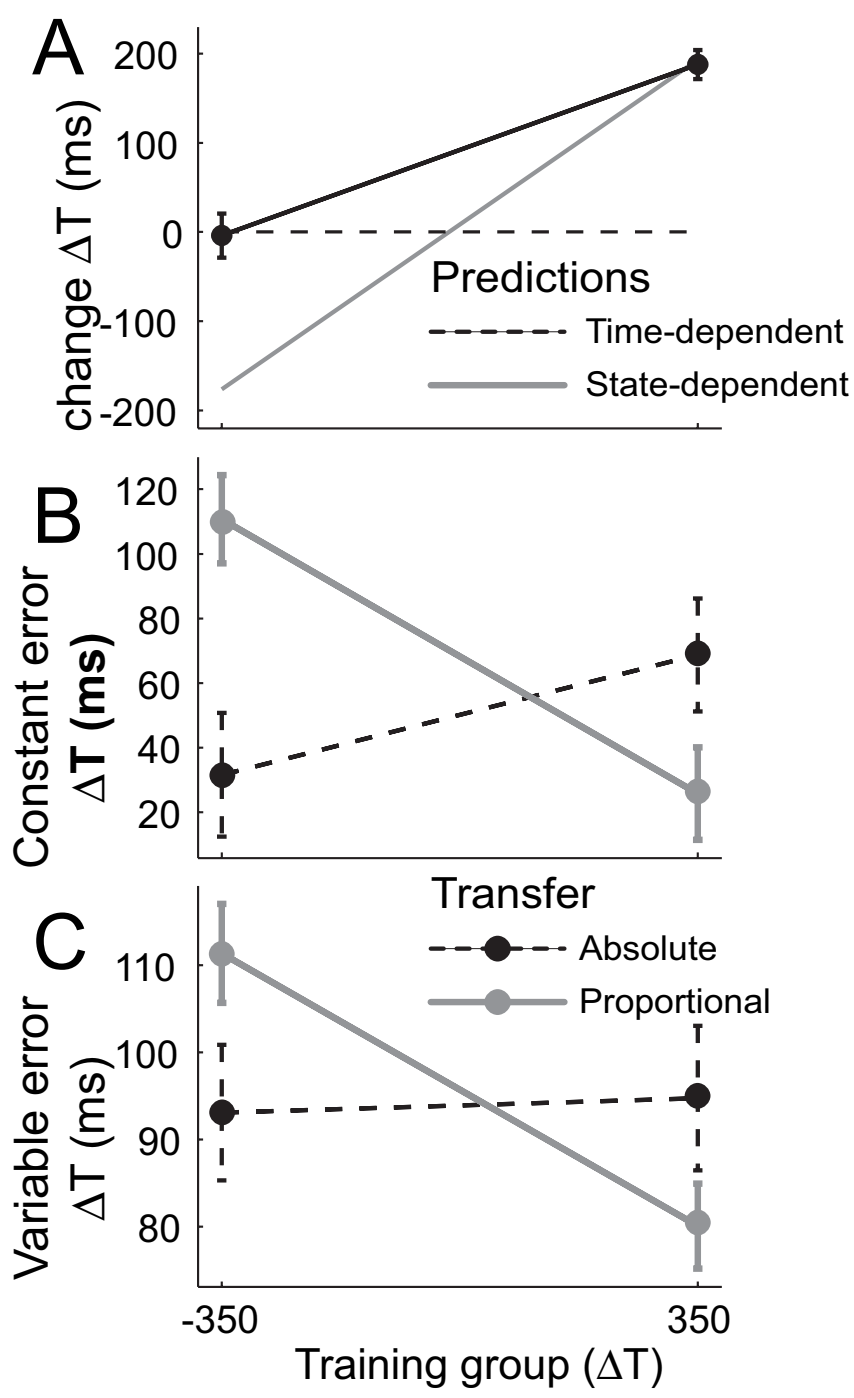

Figure 3. Result of the bilateral coordination task (experiment 2). $\boldsymbol{A}$, Change in $\Delta T$ from the last training block to the first spontaneous generalization test, and prediction of the statedependent (gray line) and time-dependent control hypothesis (dashed line). B, C, Constant error $(\boldsymbol{B})$ and variable error of $\Delta T(\boldsymbol{C})$ in the absolute and proportional transfer tests. Error bars indicate SEM.

the nonoverlapping and overlapping conditions, respectively, we found the characteristic difference in the within-person variability of $\Delta T$, with lower variability in the overlapping than in the nonoverlapping condition $\left(t_{(10)}=5.42 ; p=0.001\right)$. Note, however, that the visual error that participants saw on the screen was matched as a result of the different scaling of the visual feedback signal in the different conditions. No evidence of mirror movements was detected when only one limb moved. Participants were instructed to keep central fixation, and a control study confirmed that eye movements were rather infrequent with only small differences between the conditions (experiment 3, see Materials and Methods).

\section{Cerebellar results}

In the thumb-only and the arm-only conditions, we observed activity ipsilateral to the moving arm or thumb, respectively, in the anterior lobe (lobule V) (Fig. 4A) and the inferior cerebellum (lobule VIIIA). Both of these regions have reciprocal connections with the primary motor cortex (Kelly and Strick, 2003) and contain a representation of the ipsilateral upper extremity (Grodd et 
Table 1. Movement parameters in experiments 3 and 4

\begin{tabular}{|c|c|c|c|c|c|}
\hline & Thumb press only & Arm movement only & Overlapping & Nonoverlapping ( $-350 \mathrm{~ms}$ ) & $\begin{array}{l}\text { Nonoverlapping } \\
\text { (+700 ms) }\end{array}$ \\
\hline \multicolumn{6}{|l|}{ Experiment 3} \\
\hline Arm MT (ms): right & & $388(76)$ & $383(59)$ & $378(61)$ & \\
\hline Thumb press (N): left & $6.23(2.18)$ & & $6.10(2.24)$ & $5.90(1.80)$ & \\
\hline Relative timing (ms) & & & $374(75)$ & $-326(131)$ & \\
\hline \multicolumn{6}{|l|}{ Experiment 4} \\
\hline Arm MT (ms): left & & $373(53)$ & $367(49)$ & $357(43)$ & $355(41)$ \\
\hline Thumb press (N): right & $5.79(1.32)$ & & $6.10(1.51)$ & $5.99(1.36)$ & $5.80(1.36)$ \\
\hline Relative timing (ms) & & & $364(62)$ & $-318(98)$ & $665(65)$ \\
\hline
\end{tabular}

Mean movement time of the arm, thumb force integrated over the period of the thumb press, and the relative timing between thumb press and start of the arm movement. The data are mean and (in parentheses) average within-subject SD.

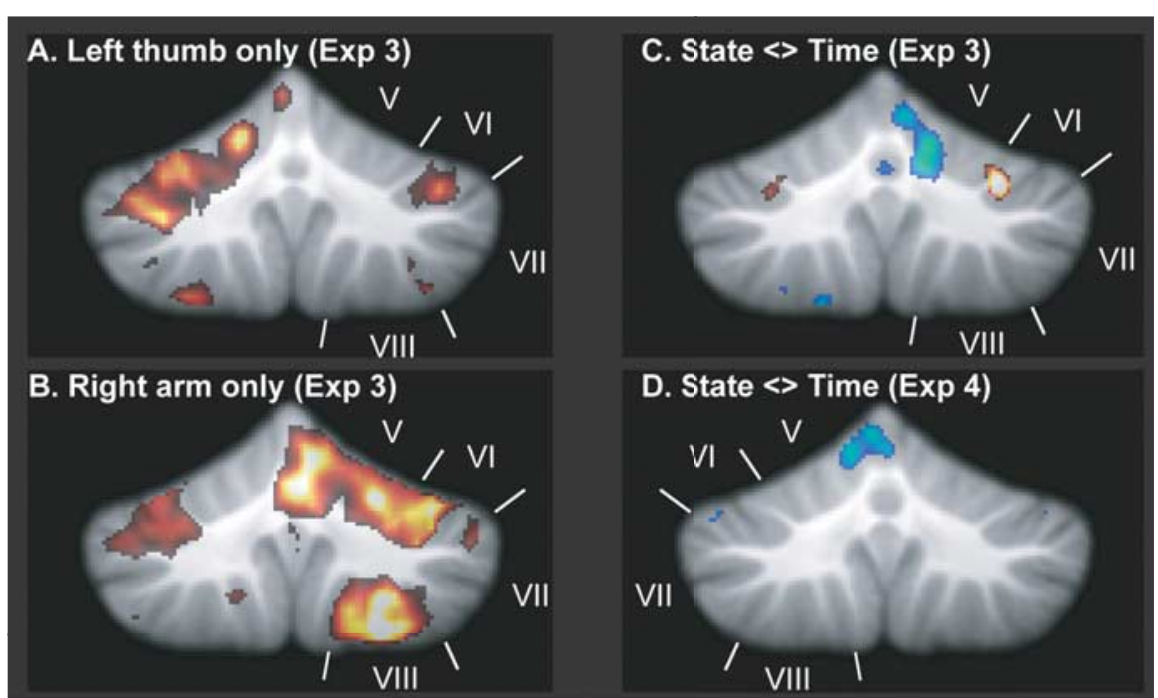

E.

Lobule V

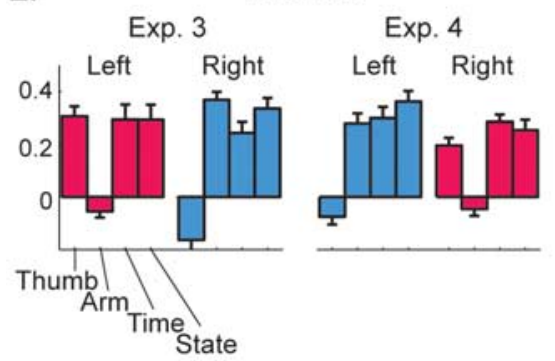

F.

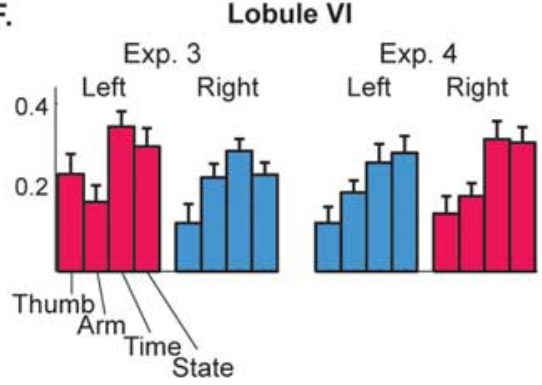

Figure 4. Cerebellar activity in experiments 3 and 4 . $A$, Thumb-only task compared with rest. $\boldsymbol{B}$, Arm-only task compared with rest. $C, D$, Contrast between state- and time-dependent control. Areas that were more activated during state-dependent control are shown in blue and areas more activated during time-dependent control are shown in red. Data are shown on coronal section of a high-resolution cerebellar atlas template (Diedrichsen, 2006). $\boldsymbol{E}, \boldsymbol{F}$, Bar graphs show the percentage of signal change in each of the four conditions (thumb-only, arm-only, time-dependent control, state-dependent) versus rest for left and right lobule $V$ and lobule VI. The hemisphere ipsilateral to the thumb movement is shown in red, the hemisphere ipsilateral to the arm movement in blue. Error bars indicate SEM. Exp., Experiment.

al., 2001). Bilateral activity in both conditions was also observed in the hemispheric portion of lobule VI. The activity caused by the arm movement was stronger than the activity caused by the thumb press, consistent with the increased demands on muscle recruitment and feedback control during reaching.

For the contrast between time- and state-dependent control, we limited our analysis to areas that were more active during any of the four tasks when compared with rest. We found more activity in the overlapping condition (state-dependent control) in the right anterior lobe of the cerebellum (Fig. 4C). This activity was quite pronounced: it was the most significant area when the analysis was performed across the whole brain and occurred in an area that showed activity elicited by the arm movement alone. The result suggested the involvement of the anterior cerebellum in the state-dependent control and, more precisely, in the estimation of the state of the arm.

We also found a small region in the right hemisphere of lobule VI that was significantly more active in the nonoverlapping condition (time-dependent control) (Fig. 4C). At lower thresholds, a small cluster could also be seen in a symmetric location in the left lobule VI.

Although these results suggested an anatomical dissociation between state- and time-dependent control in the cerebellum, there were two potential problems. First, in the nonoverlapping condition, the thumb movement preceded the arm movement, whereas the two were executed simultaneously in the overlapping condition. Therefore, it was possible that the sequence of actions rather than the requirements of control could explain the differences in BOLD signal between these two conditions. Second, compared with the anterior lobe activation in the statedependent control, the activation elicited by the time-dependent control was rather small, and we were less confident of its reliability.

\section{Experiment 4}

To address these concerns, we conducted another experiment in which two variants of the nonoverlapping task were used. In one nonoverlapping condition, the thumb press preceded the arm movement as before $(\Delta \mathrm{T}=-350 \mathrm{~ms})$, whereas in the other, the thumb press came $350 \mathrm{~ms}$ after the end of the arm movement. The behavioral results from experiment 1 had clearly shown that both of these conditions produced time-dependent control.

In this experiment, we also reversed the assignment of the task, with the left arm moving, and the right thumb producing the force pulse. If, as we hypothesized, the increased activation in the anterior lobe of the cerebellum related to the state-estimation of the arm movement, then the activity related to state-dependent control should switch to the left side of the cerebellum. 
Table 2. Cerebellar regions that showed a significant difference between state-dependent (overlapping condition), combined over experiments 3 and 4

\begin{tabular}{|c|c|c|c|c|c|c|c|c|}
\hline \multirow[b]{2}{*}{ Contrast } & \multirow[b]{2}{*}{ Area } & \multirow[b]{2}{*}{ Side } & \multirow[b]{2}{*}{ Size $\left(\mathrm{cm}^{3}\right)$} & \multirow[b]{2}{*}{ Peak $_{(21)}$} & \multirow[b]{2}{*}{$p_{\text {(cluster) }}$} & \multicolumn{3}{|c|}{ MNI (SUIT) } \\
\hline & & & & & & $x$ & $y$ & $z$ \\
\hline \multirow[t]{3}{*}{ State $>$ time } & Lobule V & Arm movement & \multirow[t]{3}{*}{3.2} & 5.95 & \multirow[t]{3}{*}{$<0.001$} & 14 & -50 & -26 \\
\hline & Lobule V & Arm movement & & 5.62 & & 8 & -58 & -16 \\
\hline & Lobule V & Arm movement & & 5.30 & & 12 & -60 & -18 \\
\hline
\end{tabular}

$p_{\text {(cluster) }}$ Significance of cluster size corrected for multiple test over the tested volume of the cerebellum at a height threshold of $p<0.001$. MNI (SUIT), Coordinates of maximum activation after alignment to the spatially unbiased infratentorial atlas template (Diedrichsen, 2006). Coordinates in this new template are congruent with coordinates after affine alignment to the MNI152 template. Please note that $x$ coordinates are absolute values because the analysis reflects location of maxima from an average analysis of left and right hemispheres.

Table 3. Cerebral regions that showed a significant or nearly significant difference between the state- and time-dependent conditions, combined over experiments 3 and 4

\begin{tabular}{|c|c|c|c|c|c|c|c|c|}
\hline \multirow[b]{2}{*}{ Contrast } & \multirow[b]{2}{*}{ Area } & \multirow[b]{2}{*}{ Side (contralateral to) } & \multirow[b]{2}{*}{ Size $\left(\mathrm{cm}^{2}\right)$} & \multirow[b]{2}{*}{ Peak $t_{(10)}$} & \multirow[b]{2}{*}{$p_{\text {(cluster) }}$} & \multicolumn{3}{|c|}{ MNI (PALS) } \\
\hline & & & & & & $x$ & $y$ & $z$ \\
\hline \multirow[t]{5}{*}{ State $>$ time } & Primary motor cortex & Arm movement & 0.41 & 5.506 & 0.003 & 34.6 & -23.4 & 56.2 \\
\hline & SMA & Arm movement & 0.32 & 4.884 & 0.017 & 6.2 & -7.3 & 56.3 \\
\hline & Dorsal premotor & Thumb movement & 0.94 & 7.251 & $<0.001$ & 27.3 & -11.1 & 57.1 \\
\hline & Dorsal premotor & Thumb movement & 0.39 & 5.137 & 0.005 & 23.0 & -7.5 & 62.9 \\
\hline & Anterior intraparietal sulcus & Thumb movement & 0.58 & 5.202 & $<0.001$ & 33.5 & -50.9 & 63.8 \\
\hline \multirow[t]{2}{*}{ Time $>$ state } & Planum temporale & Left & 0.71 & 4.705 & $<0.001$ & -52.8 & -40.2 & 20.2 \\
\hline & Insula & Left & 0.25 & 5.023 & 0.064 & -36.7 & 14.4 & 0.6 \\
\hline
\end{tabular}

$p_{\text {(cluster), }}$ Significance of cluster size corrected for multiple test over the surface of each hemispheres at a height threshold of $t_{(21)}=3.53, p<0.001$. MNI (PALS), Coordinates in MNI-space of maximum activation after alignment to the surface-based atlas (Van Essen, 2005). Note that for the contrast state $>$ time, $x$ coordinates are in absolute values, indicating the location in both left and right hemispheres.

Behavioral results

As before, the arm movement times and total force impulses of the thumb were well matched between all conditions (Table 1); none of the possible pairwise comparisons were significant (all $\left.t_{(10)}<1.86 ; p>0.09\right)$. The data on $\Delta T$ indicated that participants were able to produce the required relative timing.

\section{Cerebellar results}

The thumb-only and the arm-only conditions led to mirrorsymmetric activation patterns compared with experiment 3 , consistent with the change in hand assignment. Importantly, we again observed significantly stronger activity during state- than during time-dependent control in the anterior lobe, this time in the left lobule V. Given the switch in activity from the right to the left side in the state-dependent contrast, we combined the results from experiments 3 and 4 by averaging the two cerebellar hemispheres ipsilateral to the arm movement and the two hemispheres ipsilateral to the thumb press. In this analysis, we found only one large cluster with more activity during state- than during timedependent control, located in lobule $\mathrm{V}$ ipsilateral to the arm movement (Table 2). The results not only demonstrated a prominent role for the cerebellum in state-dependent control, but also suggested that in this task, the specific function was to estimate the state of the arm, rather than use the estimate of the state that might be generated elsewhere to control the thumb press.

An important concern was that the sequence of actions, rather than the mode of control, was responsible for the difference between conditions. Experiment 4 gave us the opportunity to test this hypothesis directly. For the anterior lobe ipsilateral to the arm movement, there was no significant differences between the two nonoverlapping conditions $\left(t_{(10)}=1.11 ; p=0.293\right)$. More importantly, the overlapping condition led to significantly more activity than either of the nonoverlapping conditions $\left(t_{(10)}=\right.$ 2.23; $p<0.026)$.

Alternatively, our results may have been caused by a nonlinearity in how the hemodynamic signals of two overlapping or nonoverlapping neural events combine. Although we do not yet fully understand how the BOLD signals of two events combine, we do not think that this can account for our results. Although the anterior lobe ipsilateral to the thumb press showed the mirror image for the activity in the arm-only and the thumb-only conditions (Fig. 4, red bar plots), it did not show a difference between the overlapping and nonoverlapping conditions.

Finally, we believe that eye movements cannot account for the differences observed here, as eye movement-related activity is typically observed in the cerebellar vermis VI/VIIa (Nitschke et al., 2005), quite distant from regions in which we found differences between the tasks here.

We did not replicate the result for more activity during timedependent control in lobule VI, as we had observed in experiment 3. Even when the results of the two experiments were combined, no significant cluster with more activity during time-dependent control was found in the cerebellum. This failure was not because of the balancing of the sequence of actions. Even when the comparison was made between the exact same conditions as in experiment 3, no difference emerged in lobule VI. Therefore, although we found robust activity in the cerebellum associated with statedependent control, we did not find robust activity associated with time-dependent control.

\section{Cortical activity (experiments 3 and 4 )}

In both experiments 3 and 4, we observed a number of dorsal motor regions that were more activated during state- than during time-dependent control. All of these regions switched cerebral hemispheres when the task assignment to the hands was switched. We quantified these observations by correlating pattern of activity difference between experiments. When we correlated the pattern of the left with the left and of the right with the right hemisphere, the correlation was much lower $(r=0.09)$, then we correlated the patterns by flipping the hemispheres with the flip in-hand assignment $(r=0.29)$. We, therefore, combined the experiments by averaging the hemispheres contralateral to the arm and thumb movements together.

In the hemisphere contralateral to the moving arm, we found a region in the primary motor cortex and the supplementary motor area (SMA) that showed significantly more activation during state-dependent control (Table 3, Fig. 5A). In the hemisphere contralateral to the thumb press, we found two areas in the dorsal premotor cortex, as well as one site in the anterior aspect of the 

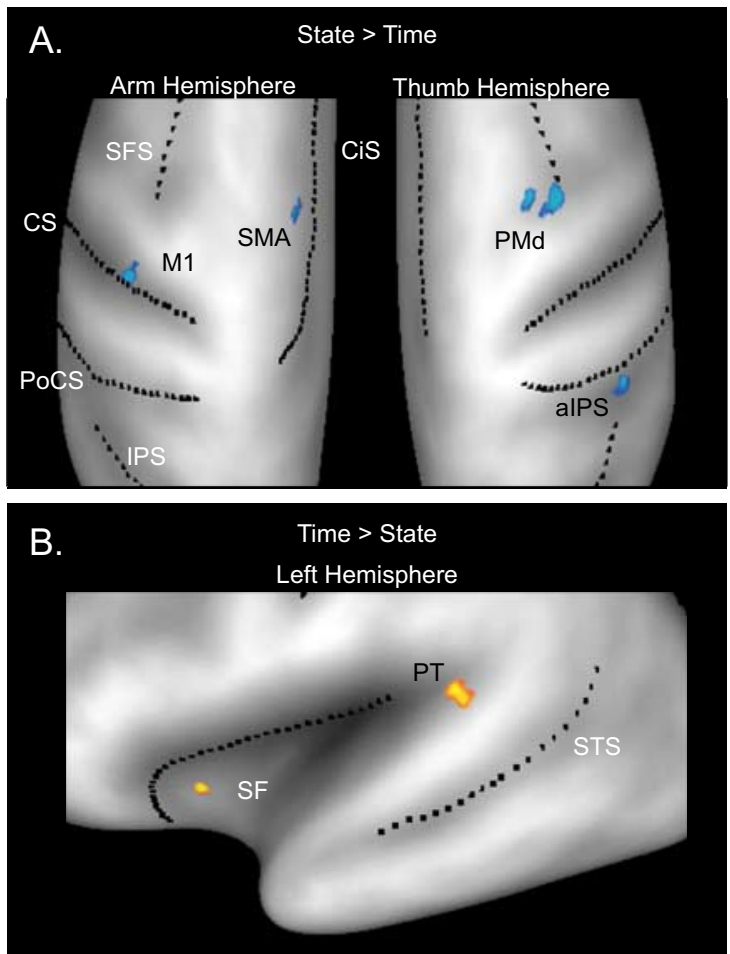

C.
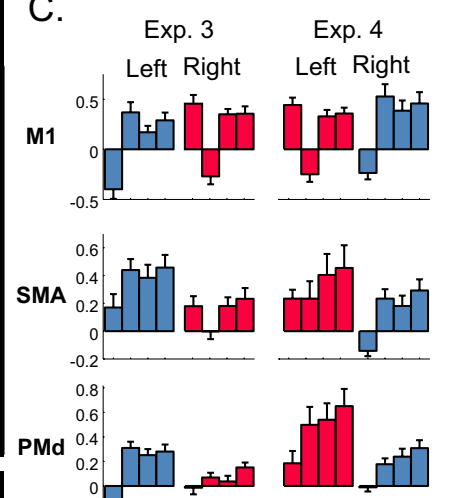

0

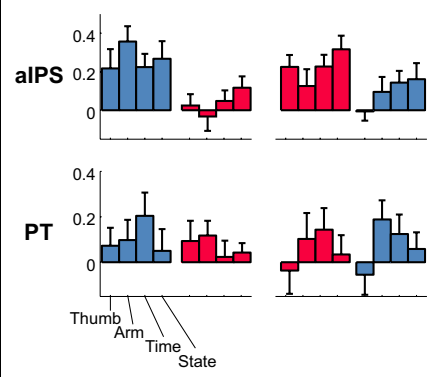

Figure 5. A, Areas of the cerebral hemispheres contralateral to the arm movement (left) and contralateral to the thumb movement (right) showing more activity during state-dependent control (blue). $\boldsymbol{B}$, Areas in the left hemisphere showing more activation during time-dependent control (red). Group analysis was performed using a surface-based atlas and activity differences were thresholded at $t_{(20)}>3.53, p<0.001$. C, Percentage of signal change versus rest for the four experimental conditions, for symmetric locations in experiments 3 and 4. The hemisphere contralateral to the arm movement is shown in blue, the hemisphere contralateral to the thumb movement is shown in red. Error bars indicate SEM. CiS, Cingulate sulcus; CS, central sulcus; IPS, intraparietal sulcus; PoCS, postcentral sulcus; SF, Sylvian fissure; SFS, superior frontal sulcus; STS, superior temporal sulcus; M1, primary motor cortex; SMA, supplementary motor area; PMd, dorsal premotor area; alPS, anterior intraparietal sulcus; PT, planum temporale.

intraparietal sulcus. None of these regions showed an effect of the sequence of movements in experiment $4\left(t_{(10)}<1.35 ; p>0.2\right)$, except for the dorsolateral premotor cortex that showed more activity when the arm movement occurred before the thumb press $\left(t_{(10)}=3.353 ; p=0.007\right)$. However, this region was significantly more activated during state-dependent control than during time-dependent control with either sequence of actions $\left(t_{(10)}>2.85 ; p<0.017\right)$.

Within the same analysis, we also searched for regions that were more activated during time- than during state-dependent control. The only significant cluster was in the planum temporale (BA 42) on the side contralateral to the arm movement $(p<$ 0.001 , corrected for multiple tests). However, inspection of the activity in this region and the symmetric area in the other hemisphere (Fig. $5 C$, bar plots) revealed that this difference was always more pronounced in the left hemisphere. To quantify this observation, we performed an ANOVA with the factors experiment, side (left vs right), and condition (state vs time). Only the planum temporale, but not regions that were more active during statedependent control, showed a significant hemisphere by condition interaction $\left(F_{(1,20)}=9.14 ; p=0.007\right)$. We therefore combined the experiments by averaging the two left and the two right hemispheres together (Fig. 5B, Table 3). This revealed the planum temporale even more clearly than in the last analysis and also showed a marginally significant region in the insula/frontal operculum. None of our analyses found significant differences between time- and state-dependent control in the thalamus or basal ganglia.
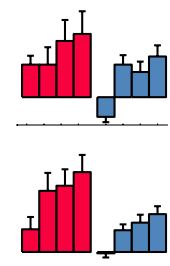

The BOLD signal in cerebellar lobule $\mathrm{V}$ ipsilateral to the arm movement and M1 contralateral to the arm movement was lower in both state- or time-dependent control conditions than during arm movements alone. However, it has to be taken into account that the BOLD signal in these regions is suppressed by the thumb movement. Until we have a precise model of how the BOLD signal of two single events combine when the two events occur in a small time window (which is likely a more complicated nonlinear relationship), it is not meaningful to compare the state- and time-dependent coordination to the single events. Therefore, we use here the comparison between two conditions, in which the same events occurred within a short time window (compared with the window of the hemodynamic response function).

In summary, cortical activity associated with state-dependent control was in the M1 and SMA in the hemisphere contralateral to the arm and in the dorsal premotor area and anterior intraparietal sulcus contralateral to the thumb. However, cortical activity associated with timedependent control was always in the left hemisphere in areas along the Sylvian fissure.

\section{Discussion}

We found a behavioral and neural dissociation between the timing and the coordination of movements. When the arm movement and the thumb press were separated by a small temporal gap, participants learned to trigger the components based on an internal estimate of time. When the two movement components overlapped, the participants learned to produce the thumb press based on the state of the arm movement. These results were independent of whether feedback was given in absolute or relative time.

This indicates that state-dependent control is the default mode for the nervous system when two temporally overlapping motor components need to be coordinated (Cordo, 1990; Conditt and Mussa-Ivaldi, 1999). Because the position, velocity, and joint angles of the arm changed between learning and generalization testing, a system that would have relied on one of these states in isolation would not have shown the observed pattern of generalization. Rather, the underlying state estimate must have represented a more abstract variable, such as the percentage of the movement completed. Also, the state estimate cannot be based solely on sensory feedback. In the $150 \mathrm{~ms}$ condition of our experiment, the thumb press starts only $50 \mathrm{~ms}$ after the arm movement begins, too short to initiate or adjust a movement plan based on sensory feedback (Hore et al., 1999). Therefore, movement coordination must be based on a predictive state estimate of the moving limb. Such a state estimate would combine a forward-model prediction based on an efference copy of the movement with sensory feedback (Wolpert et al., 1998; Vaziri et al., 2006), while internally correcting for sensory delays (Miall et al., 1993).

We found that the anterior lobe of the cerebellum (lobule V) 
was consistently more activated during state-dependent control than time-dependent control. This region of the cerebellum contains a motor homunculus (Grodd et al., 2001) and is part of a recurrent loop with the contralateral primary motor cortex (Kelly and Strick, 2003). Increases in BOLD signal in the cerebellar cortex reflect increased climbing and/or parallel fiber input (Caesar et al., 2003). This coordination-related activity was always ipsilateral to the moving arm, suggesting that during statedependent control, the cerebellum provides an estimate of the state of the arm rather than assisting control of the thumb movement. This hypothesis is consistent with results from a recent imaging study that investigated cerebellar activity during a task in which eye movements guided hand movements and vice versa (Miall and Jenkinson, 2005). Increased input to the cerebellar cortex was found in regions related to the leading effector, the effector whose state-estimate was used to generate the commands to the other effector. Together, the robust cerebellar activity during state-dependent coordination is likely a reflection of the participation of this structure in predicting the state of the moving effector. Given our cortical results, we hypothesize that this function would be achieved in a corticocerebellar loop with the primary motor cortex and the SMA contralateral to the arm movement. The state estimate would then be transferred to the other hemisphere, where other areas would influence the control of the thumb based on this estimate. Our results indicate an involvement of the anterior aspect of the intraparietal sulcus as well as the dorsal premotor cortex, both of which are involved for control of finger movements and forces for grasping (Culham et al., 2003; Davare et al., 2006).

When movements were separated by a small temporal gap, participants generated motor commands based on an estimate of absolute time. This process was associated with activation in the left hemisphere, most prominently the planum temporale (BA 42). This area has been shown to be activated by internal speech (Buchsbaum et al., 2005), suggesting that participants may have solved the task of time-dependent control by using an internal rhythmic representation to bridge the time between the two movements. Indeed, although participants were instructed not to produce any speech during the task, about half of the participants reported they had, at least in early stages of training, used a covert pattern such as "press-wait-move" to perform the nonoverlapping task. In contrast to areas active during state-dependent control, which switched sides with a change in hand assignment, activity during time-dependent control was always stronger in the left planum temporale regardless of hand assignment.

We did not find robust activity in the cerebellum during timedependent control. In experiment 3, we found a small area in the right lobule VI, but this result was not reproduced in experiment 4 and was also not present when we pooled the data from both experiments. Activity in the hemispheric portions of lobule VI or VII has been noted in a number of studies that have compared the production or perception of difficult noninteger rhythms to isochronous rhythms (Sakai et al., 1999; Kawashima et al., 2000; Ramnani and Passingham, 2001; Dreher and Grafman, 2002; Harrington et al., 2004; Bengtsson et al., 2005; Chen et al., 2005; Jantzen et al., 2005; Xu et al., 2006). Such an involvement may account for cerebellar deficits in the production of timed motor commands, especially when the movements are discontinuous [i.e., contain short phases in which no movement occurs (Spencer et al., 2003)]. However, compared with the state-dependent control condition, time-dependent control of movements did not produce robust activity in the cerebellum.

In summary, we found that the temporal overlap of two movement components predicted whether the brain controlled a task via a state-dependent or a time-dependent process. When the two components temporally overlapped, the brain used a state-dependent control process in which the state of one action affected the control of the other action. When the two components did not have a temporal overlap, the brain used a timedependent control process in which initiation of each component relied on an internal representation of time. When we compared these two control modes directly, we found that only statedependent control produced robust activation of the cerebellum. This activity was always on the side ipsilateral to the arm movement, suggesting a role for the cerebellum in state estimation for coordination (Paulin, 2005; Ramnani, 2006).

\section{References}

Ashburner J, Friston KJ (1999) Nonlinear spatial normalization using basis functions. Hum Brain Mapp 7:254-266.

Bastian AJ, Martin TA, Keating JG, Thach WT (1996) Cerebellar ataxia: abnormal control of interaction torques across multiple joints. J Neurophysiol 76:492-509.

Bengtsson SL, Ehrsson HH, Forssberg H, Ullen F (2005) Effectorindependent voluntary timing: behavioural and neuroimaging evidence. Eur J Neurosci 22:3255-3265.

Buchsbaum BR, Olsen RK, Koch PF, Kohn P, Kippenhan JS, Berman KF (2005) Reading, hearing, and the planum temporale. NeuroImage 24:444-454.

Caesar K, Gold L, Lauritzen M (2003) Context sensitivity of activitydependent increases in cerebral blood flow. Proc Natl Acad Sci USA 100:4239-4244.

Chen JL, Penhune VB, Zatorre RJ (2005) Tapping in synchrony to auditory rhythms: effect of temporal structure on behavior and neural activity. Ann NY Acad Sci 1060:400-403.

Conditt MA, Mussa-Ivaldi FA (1999) Central representation of time during motor learning. Proc Natl Acad Sci USA 96:11625-11630.

Cordo PJ (1990) Kinesthetic control of a multijoint movement sequence. J Neurophysiol 63:161-172.

Culham JC, Danckert SL, DeSouza JF, Gati JS, Menon RS, Goodale MA (2003) Visually guided grasping produces fMRI activation in dorsal but not ventral stream brain areas. Exp Brain Res 153:180-189.

Davare M, Andres M, Cosnard G, Thonnard JL, Olivier E (2006) Dissociating the role of ventral and dorsal premotor cortex in precision grasping. J Neurosci 26:2260-2268.

Diedrichsen J (2006) A spatially unbiased atlas template of the human cerebellum. NeuroImage 33:127-138.

Diedrichsen J, Shadmehr R (2005) Detecting and adjusting for artifacts in fMRI time series data. NeuroImage 27:624-634.

Diedrichsen J, Hashambhoy YL, Rane T, Shadmehr R (2005) Neural correlates of reach errors. J Neurosci 25:9919-9931.

Dreher JC, Grafman J (2002) The roles of the cerebellum and basal ganglia in timing and error prediction. Eur J Neurosci 16:1609-1619.

Friston KJ, Worsley KJ, Frackowiak RSJ, Mazziotta JC, Evans AC (1994) Assessing the significance of focal activations using their spatial extent. Hum Brain Mapp 1:214-220.

Gerwig M, Dimitrova A, Kolb FP, Maschke M, Brol B, Kunnel A, Boring D, Thilmann AF, Forsting M, Diener HC, Timmann D (2003) Comparison of eyeblink conditioning in patients with superior and posterior inferior cerebellar lesions. Brain 126:71-94.

Gibbon J, Malapani C, Dale CL, Gallistel CR (1997) Toward a neurobiology of temporal cognition: advances and challenges. Curr Opin Neurobiol 7:170-184.

Grodd W, Hulsmann E, Lotze M, Wildgruber D, Erb M (2001) Sensorimotor mapping of the human cerebellum: fMRI evidence of somatotopic organization. Hum Brain Mapp 13:55-73.

Harrington DL, Boyd LA, Mayer AR, Sheltraw DM, Lee RR, Huang M, Rao SM (2004) Neural representation of interval encoding and decision making. Brain Res Cogn Brain Res 21:193-205.

Hore J, Watts S (2005) Timing finger opening in overarm throwing based on a spatial representation of hand path. J Neurophysiol 93:3189-3199.

Hore J, Ritchie R, Watts S (1999) Finger opening in an overarm throw is not triggered by proprioceptive feedback from elbow extension or wrist flexion. Exp Brain Res 125:302-312. 
Ivry RB (1996) The representation of temporal information in perception and motor control. Curr Opin Neurobiol 6:851-857.

Ivry RB, Keele SW (1989) Timing functions of the cerebellum. J Cogn Neurosci 1:136-152.

Ivry RB, Spencer RM, Zelaznik HN, Diedrichsen J (2002) The cerebellum and event timing. Ann NY Acad Sci 978:302-317.

Jantzen KJ, Steinberg FL, Kelso JA (2005) Functional MRI reveals the existence of modality and coordination-dependent timing networks. NeuroImage 25:1031-1042.

Karmarkar UR, Buonomano DV (2003) Temporal specificity of perceptual learning in an auditory discrimination task. Learn Mem 10:141-147.

Kawashima R, Okuda J, Umetsu A, Sugiura M, Inoue K, Suzuki K, Tabuchi M, Tsukiura T, Narayan SL, Nagasaka T, Yanagawa I, Fujii T, Takahashi S, Fukuda H, Yamadori A (2000) Human cerebellum plays an important role in memory-timed finger movement: an fMRI study. J Neurophysiol 83:1079-1087.

Kelly RM, Strick PL (2003) Cerebellar loops with motor cortex and prefrontal cortex of a nonhuman primate. J Neurosci 23:8432-8444.

Mauk MD, Medina JF, Nores WL, Ohyama T (2000) Cerebellar function: coordination, learning or timing? Curr Biol 10:R522-525.

Meegan DV, Aslin RN, Jacobs RA (2000) Motor timing learned without motor training. Nat Neurosci 3:860-862.

Miall RC, Jenkinson EW (2005) Functional imaging of changes in cerebellar activity related to learning during a novel eye-hand tracking task. Exp Brain Res 166:170-183.

Miall RC, Weir DJ, Wolpert DM, Stein JF (1993) Is the cerebellum a Smith predictor? J Mot Behav 25:203-216.

Nitschke MF, Arp T, Stavrou G, Erdmann C, Heide W (2005) The cerebellum in the cerebro-cerebellar network for the control of eye and hand movements-an fMRI study. Prog Brain Res 148:151-164.

Paulin MG (2005) Evolution of the cerebellum as a neuronal machine for Bayesian state estimation. J Neural Eng 2:S219-S234.

Pruessmann KP, Weiger M, Scheidegger MB, Boesiger P (1999) SENSE: sensitivity encoding for fast MRI. Magn Reson Med 42:952-962.
Ramnani N (2006) The primate cortico-cerebellar system: anatomy and function. Nat Rev Neurosci 7:511-522.

Ramnani N, Passingham RE (2001) Changes in the human brain during rhythm learning. J Cogn Neurosci 13:952-966.

Ramnani N, Toni I, Passingham RE, Haggard P (2001) The cerebellum and parietal cortex play a specific role in coordination: a PET study. NeuroImage 14:899-911.

Sakai K, Hikosaka O, Miyauchi S, Takino R, Tamada T, Iwata NK, Nielsen M (1999) Neural representation of a rhythm depends on its interval ratio. J Neurosci 19:10074-10081.

Seidler RD, Noll DC, Thiers G (2004) Feedforward and feedback processes in motor control. NeuroImage 22:1775-1783.

Spencer RM, Zelaznik HN, Diedrichsen J, Ivry RB (2003) Disrupted timing of discontinuous but not continuous movements by cerebellar lesions. Science 300:1437-1439.

Timmann D, Watts S, Hore J (1999) Failure of cerebellar patients to time finger opening precisely causes ball high-low inaccuracy in overarm throws. J Neurophysiology 82:103-114.

Van Essen DC (2005) A population-average, landmark- and surface-based (PALS) atlas of human cerebral cortex. NeuroImage 28:635-662.

Van Essen DC, Dickson J, Harwell J, Hanlon D, Anderson CH, Drury HA (2001) An integrated software system for surface-based analyses of cerebral cortex. J Am Med Inform Assoc 41:1359-1378.

Vaziri S, Diedrichsen J, Shadmehr R (2006) Why does the brain predict sensory consequences of oculomotor commands? Optimal integration of the predicted and the actual sensory feedback. J Neurosci 26:4188-4197.

Wenderoth N, Debaere F, Sunaert S, Swinnen SP (2005) The role of anterior cingulate cortex and precuneus in the coordination of motor behaviour. Eur J Neurosci 22:235-246.

Wolpert DM, Miall RC, Kawato M (1998) Internal models in the cerebellum. Trends Cogn Sci 2:313-321.

Xu D, Liu T, Ashe J, Bushara KO (2006) Role of the olivo-cerebellar system in timing. J Neurosci 26:5990-5995. 